\title{
CONTROL OF A DRONE WITH BODY GESTURES
}

\author{
Gio, Nicolas (1,2); \\ Brisco, Ross (1); \\ Vuletic, Tijana (1) \\ 1: DMEM, University of Strathclyde; \\ 2: Arts et Métiers ParisTech
}

\begin{abstract}
Drones are becoming more popular within military applications and civil aviation by hobbyists and business. Achieving a natural Human-Drone Interaction (HDI) would enable unskilled drone pilots to take part in the flying of these devices and more generally easy the use of drones. The research within this paper focuses on the design and development of a Natural User Interface (NUI) allowing a user to pilot a drone with body gestures. A Microsoft Kinect was used to capture the user's body information which was processed by a motion recognition algorithm and converted into commands for the drone. The implementation of a Graphical User Interface (GUI) gives feedback to the user. Visual feedback from the drone's onboard camera is provided on a screen and an interactive menu controlled by body gestures and allowing the choice of functionalities such as photo and video capture or take-off and landing has been implemented. This research resulted in an efficient and functional system, more instinctive, natural, immersive and fun than piloting using a physical controller, including innovative aspects such as the implementation of additional functionalities to the drone's piloting and control of the flight speed.
\end{abstract}

Keywords: Human-Drone Interaction, Natural User Interface, Design engineering, Design for interfaces, User centred design

\section{Contact:}

Gio, Nicolas Clément

University of Strathclyde

DMEM

France

nicolas.gio@gadz.org

Cite this article: Gio, N., Brisco, R., Vuletic, T. (2021) 'Control of a Drone with Body Gestures', in Proceedings of the International Conference on Engineering Design (ICED21), Gothenburg, Sweden, 16-20 August 2021. DOI:10.1017/ pds. 2021.76 


\section{INTRODUCTION}

Human-Robot Interaction (HRI) is a field of study dedicated to understanding, designing, and evaluating robotic systems for use by or with humans (Goodrich \& Schultz, 2007). It is becoming increasingly important to today's society, given the increasing use of robots in domestic and professional environments (Andujar \& Tezza, 2019). Drones are becoming smaller and more agile, introducing the research field of Human-Drone Interaction (HDI). Once used exclusively by the military they are now being used by the general public for entertainment or photographic purposes (Andujar \& Tezza, 2019).

Traditionally a drone is piloted using PC hardware for HRI, including a controller (games controller) or a joystick. There has been more attention to HDI as a suitable interface for natural and intuitive control in recent years, which requires training and concentration. Designing an alternative interface to a controller or joystick, which would be easy to use, precise and intuitive would improve the piloting experience and widen the scope and number of users.

Research on modern user interfaces, called Natural User Interfaces (NUI) is prolific and encompasses different approaches and technologies for drone control. NUI allow the user to control their drone through speech, touch, gaze, gestures, and brain-computer interfaces. Studies such as those by Cauchard, et al. (2015) and Abtahi, et al. (2017) show that, in the absence of any instruction given to users, the first choice for interaction with a drone is gestural interaction piloting, as it allows natural and intuitive interaction. It is possible to split body gesture interaction into two categories: systems using wearable devices with embedded sensors and systems collecting user information via external sensing. The latter, essentially carried out by depth camera systems, is non-intrusive and avoids any physical constraints on the user, making it possible to use intuitive movements. Body gesture interaction will be further discussed in the literature review section.

The development of a body gesture interactive system to control a drone aims to be natural, immersive and easy to use. This can revolutionise piloting possibilities for personal and professional use, contribute to the societal transformation in relation to drone use, and change many industry sectors such as transport, culture, engineering practices, and design for high-risk environments.

This paper documents the design of a NUI using body gestures to pilot a drone. A Microsoft Kinect depth camera was used to detect the gestures and programming allowed the gesture input to be read, understood, and controls sent to the drone. Considerations for the piloting of a drone are investigated for the professional sector and the general public. Aiming to be intuitive and easy to use, the NUI is designed for both experienced and inexperienced users. This research could consequently contribute to the standardisation of new HDI, and generally be a step forward for the use of NUI in the field of HDI.

\section{LITERATURE REVIEW}

HRI benefits in methodologies, design principles, and computing metaphors from the field of HumanComputer Interaction (HCI) (Toumi, et al., 2014). HDI is a field of study of HRI that focuses on the understanding, designing, and evaluating drone systems intended for use by or with human users, with the unique characteristic of drones to move freely in 3D space making interaction innovative (Dante Tezz, 2019). Often, natural interaction is achieved by use of gestures: Hand Gesture Interaction or Body Gesture Interaction.

The interaction is captured either using wearable devices or unintrusive systems. For piloting based on hand movements, a common approach is to directly map the hands' orientations and positions and convert them into pitch, roll, and yaw rates (Suarez-Fernandez, et al., 2016). Research using glovebased devices for the collection of hand movement information has been carried out since the 1990s (Baudouin-Lafon, 1993), until recent times (Stone, 2017). Electromyography (EMG) sensors have also been researched, for example in the work of Stoica, et al. (2014), who developed a NUI using EMG signals from the forearm. Research has been conducted into body controlled HDI, investigating the effect of providing haptic feedback to the pilot in the physical system he is lying in (Ars Electronica, 2020, and Cherpillod, et al., 2016). Research, such as the Flyjacket from Rognon, C. et al., (2018), implements inertial measurement units on the user's body to collect the gestures. All these systems using wearable devices allow natural piloting but need to be worn, so they are intrusive and physically constraining for the user.

Unintrusive systems are explored to counter these constraints and are often based on vision systems. Researchers have been successful in creating single-camera systems, allowing the use of the drone's embedded camera. Hasanuzzaman, et al. (2004) and Natarajan, et al., (2018) conducted such projects 
with hand gesture piloting. The solution, however, did not allow very efficient and accurate analysis of hand gestures. Similar was found for body piloting, and the conclusion was that gesture recognition depends on the quality of the onboard video camera (Sun, et al. 2018). It also implies the drone must fly close to the user, which represents a constraint of use (Sun, et al. 2018). To increase precision, the research focus has moved on to depth cameras. Leap Motion Controller (LMC), a technology that was launched in 2012 was designed particularly for hand recognition (Ultraleap, 2019). It allows a very accurate recognition, with a slow latency, and Sakar, et al. (2016) used it to develop a NUI for drone piloting. LMC capabilities introduce certain constraints. The system must be located below the hands, in close proximity as the detection range is specified at a maximum of $80 \mathrm{~cm}$. Kinect device developed by Microsoft (2012) is also commonly used. It enables the recognition of the whole-body movements in real-time with great precision (Shotton, et al., 2010), and the Software Development Kit (SDK) provided supports easy development. Work by Sanna, et al. (2013) and Mashood, et al. (2015) both provide examples of a Kinect-based NUI used to control a drone. Sanna, et al. (2013) reported a high piloting precision compared with a controller, but with an average mission completion time of about twice as long. Both, Sanna, et al. (2013) and Mashood, et al. (2015) did not consider other functionalities required to fully control a drone such as being able to control a menu system instructing the drone to take a photo or videos, or crucially for piloting functionalities such as the control of flight speed.

Many different approaches could be taken for a mapping between body gestures and drone commands during the NUI design. Pfeil, et al. (2013) conducted a study exploring upper body 3D spatial interaction techniques for drones. Several interaction metaphors were considered, such as using the arms as game controller sticks or asking the user to move the drone as though it was grasped in their hands. The different metaphors were evaluated through a survey conducted with 14 participants, with various backgrounds and experiences, who had to complete a flying mission and evaluate each technique. The evaluation criteria were: Natural, Confusion, Comfortable, Fun, Like, Easy, Frustration, Expectation. Its outcomes highlight the strengths and weaknesses of the considered metaphors, and result in a First Person technique, which requires the user to imitate an aircraft. The movement of the upper body is linked to the movement of the drone, which appears natural and very easy to understand. However, there are not many studies present in the field where gestures were explored to the same level of detail, and a gesture vocabulary that supports all features and functions required to successfully control a drone would align research efforts across the field.

Using body gesture interaction and the depth camera to pilot a drone is a promising solution, however, more research needs to be conducted to enhance NUI, by improving the mapping between body gestures and drone commands to design an intuitive, ergonomic, and natural system. At present and for all NUIs reviewed, speed control is not implemented or implemented to a limited extent. Additionally, the published research only considers the drone's piloting aspect. Additional functionalities such as taking pictures, taking videos, or allowing the possibility to add other functions would represent an improvement for the interface and its functionality. These gaps will be addressed in this paper.

\section{RESEARCH DESIGN AND METHODOLOGY}

This research aims to develop a natural, ergonomic, and intuitive interaction system for the control of a drone using human body gestures in place of a physical controller. The envisioned system consists of a Microsoft Kinect for gesture detection, a PC and a screen for processing and visualisation and a Tello Drone from DJI (2020). Users perform body gestures, which are interpreted by the system, and appropriate commands are then sent to a drone. Figure 1 presents a high-level description of the NUI, including the elements as well as the data flow.

The Kinect located in front of the user recreates a skeleton of joints in the 3D space and sends this data to the computer. The computer processes this data through a Python algorithm and then sends the piloting commands to the drone. If the drone is not close enough to be seen, visual feedback is also possible for the user via the screen. The video of the embedded camera, the battery level, the Kinect view, and an interactive menu that will be presented later can be displayed on the screen in front of the user. The interface is transparent to the user, and do not need to use a joystick or any physical component. The interface exploits intuitive actions related to natural human behaviour, i.e. body movements, without adding excessive mental or physical strain to the user. The outlined approach is a Natural User Interface (NUI), which allows for seamless and unintrusive interaction between humans and machines. 


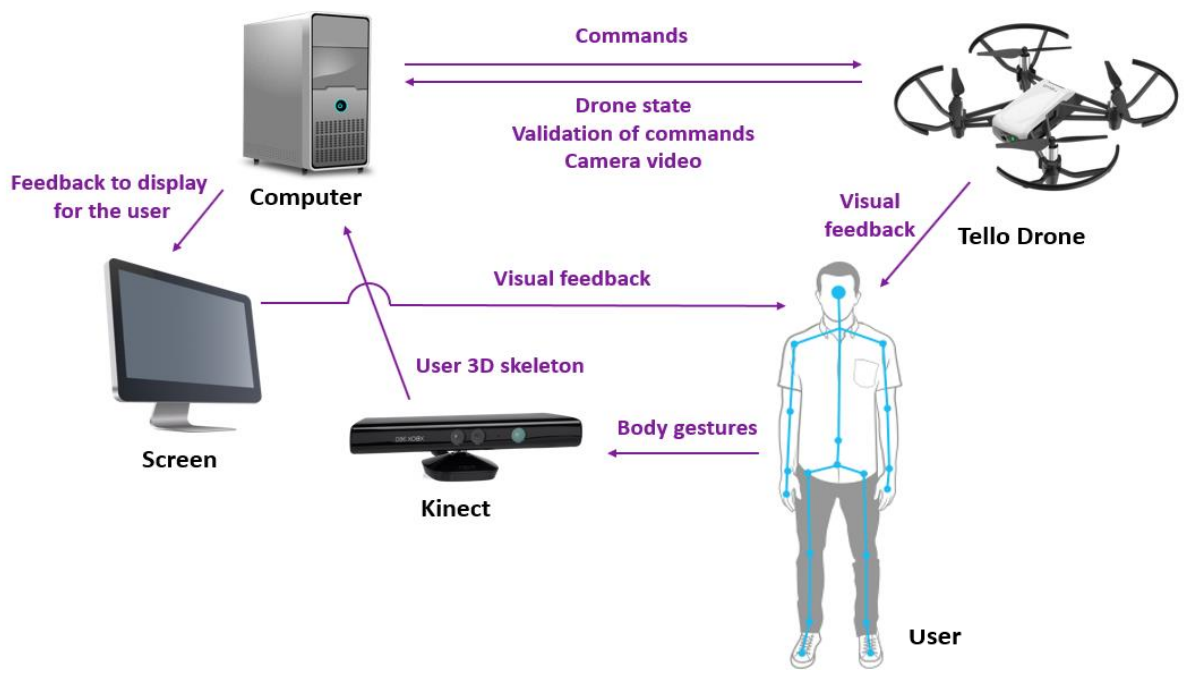

Figure 1. Structure and data flow chart of the NUI

The technology used here is for demonstration to investigate gesture control and is not necessarily the final technology that will be used. The prototype experimental setup is used for research purposes and is suitable for the capture of gestures.

\subsection{Mapping body gestures to drone commands}

Three directions (Up/Down; Forward/Backward; Right/Left) and one rotation (around a vertical axis) need to be considered to control a drone's flight. Thus, a mapping between body gestures and drone commands must be designed. The spatial interaction metaphor needs to consider users' arm fatigue, intuitive and natural aspects, speed control, and recognition accuracy.

The selected controls are presented in Table 1. They emulate first-person flying allowing for an intuitive experience. The first-person interaction technique was used as inspiration in the design of a natural, intuitive, and clear mapping between the user's body and the drone command (Pfeil, et al., 2013). Use of upper body through torso rotation arm and hand movements for control of drone was chosen in the work reported in this paper, as it corresponded well with the first-person interaction technique and allowed detection of motions relative to the size of each individual user body, making the system more ergonomic. Hand and arm gestures have been extensively researched since the $80 \mathrm{~s}$ and acknowledged as a natural and intuitive means of interaction between humans and computerised systems (Rautaray and Agrawal, 2015, Al-Shamayleh et al., 2018, Pisharady and Saerbeck, 2015).

Table 1. Correspondence between body postures and commands for the drone

\begin{tabular}{|c|c|c|c|c|c|}
\hline Command & Forward & Backward & Right / Left & $\begin{array}{c}\text { Rotation Right / } \\
\text { Left }\end{array}$ & Up / Down \\
\hline Movement & $\begin{array}{c}\text { Raise both arms } \\
\text { outwards }\end{array}$ & $\begin{array}{c}\text { Cross arms } \\
\text { in front of } \\
\text { body }\end{array}$ & $\begin{array}{c}\text { Lean Right / } \\
\text { Left }\end{array}$ & $\begin{array}{c}\text { Torso rotation to the } \\
\text { Right / Left }\end{array}$ & $\begin{array}{c}\text { Bend Backward } \\
\text { / Forward }\end{array}$ \\
\hline $\begin{array}{c}\text { Body } \\
\text { posture }\end{array}$ & &
\end{tabular}

Different aspects are considered in this research to improve the user experience. Raising the arms represents the main source of fatigue encountered when using gestures to control a robot. To reduce fatigue and consider the human factors and ergonomics, the neutral position corresponds to a straight standing position with the arms alongside the body. Arms are required to be raised only to pilot the drone to move forward or backwards. Backwards command is certainly less natural than the others, 
but this choice was made as it is also the least used command in drone piloting and is consistent with the Forward command. The Up and Down controls are inspired by the movements when piloting an aeroplane: pulling/leaning backwards the aeroplane handle for $U p$ and forwards for Down.

\subsection{Movement recognition}

The data collected by the Kinect are processed to calculate the angle values of the body to recognise the movements. The use of angles allows relative values, which do not depend on the user's distance from the Kinect or on their size. This gives the user more flexibility in space and allows them to position themselves wherever they want as long as they are within the Kinect's detection zone. Above all, the chosen design, which is not based on lengths and distances but on angles of movement, as can be seen in Table 1, considers the users' physical differences and thus works with everyone, large or small.

A threshold value, which will be chosen through practical experiments, will make it possible to confirm that the angle created is desired and that the command related to this angle must be executed.

A skeleton composed of 16 joints is retrieved from the Microsoft Kinect. The different joints are: Wrist L/R, Elbow L/R, Shoulder L/R/Centre, Head, Spine, Hip L/R/Centre, Knee L/R and Foot L/R. The different angles for each command are presented in Table 1 in red and are calculated in real-time geometrically. As an example, the geometrical calculation for the angle relative to the Right/Left command is shown in Equation 1.

$$
\begin{aligned}
& \qquad \varphi=\arctan \left(-\left(\frac{x 2-x 1}{y 2-y 1}\right)\right) \\
& \text { With } 1 \text { designing the joint "Shoulder Centre" and } 2 \text { for "Spine" }
\end{aligned}
$$

A discussion is made on the Forward and Backward commands as they involve two angles: $\theta r$ and $\theta l$. To increase accuracy and not to detect an undesired command, the minimum value will be retained and used to recognise the movement. Thus, a $\theta$ value, calculated as below, will be used.

$$
\theta=\min (|\theta r|,|\theta l|)
$$

Once all the angles have been calculated, they are used to deduce the user's gesture and therefore the desired command. Threshold values are chosen through a user survey and the desired command will be detected once an angle value is higher than the threshold.

\subsection{Speed control}

The possibility to control the drone's flight speed is necessary to fully control a drone and is a significant gap in previous research projects identified via the literature review. Thus, a significant objective of this research was to integrate this functionality without negatively impacting the piloting.

Considering that the natural and intuitive aspects are essential in this research, the chosen solution for controlling the drone's speed is to use the amplitudes of the body gestures and convert them into velocity. A more pronounced movement (e.g. a larger angle of arm raise, torso lean or torso twist) will correspond to a higher speed command. Thus, instead of the user's gesture controlling only the drone command and, therefore, its position, it controls the command as well as the associated speed. An example is shown in Figure 2. On the left side, the angle $\varphi$ of inclination of the user is small, which will generate a low lateral velocity to the right of the drone, whereas on the right side, the value of the angle being larger, the lateral velocity will also be higher. To do this, for each command, two trigger values of amplitude need to be chosen. They correspond to the angles from which the commands are detected and the maximum amplitude values that will represent the highest speed commands. The trigger values are chosen to allow the user to have a certain freedom of neutral position without an unwanted command being detected. The maximum amplitude values should represent a large range of motion but should not be excessive and not physically unpleasant or strenuous. These trigger values were chosen based on a user survey and are between 10 and 29 degrees for the minimum values, and between 29 and 47 degrees for the maximum values.

The amplitude interval must be related to the speed interval. It is possible to realise speed steps, with, for example, three possible speeds for one control. However, to make the control as natural, fluid, and pleasant as possible, a method of continuous speed control has been thought up. Thus, a bijection between amplitude and speed will be made between the range of amplitude of the movement and the desired speed range. Figure 3 and equation (4) present the linear regression and the velocity formula allowing to calculate, for all the commands, the velocity associated with each amplitude. 


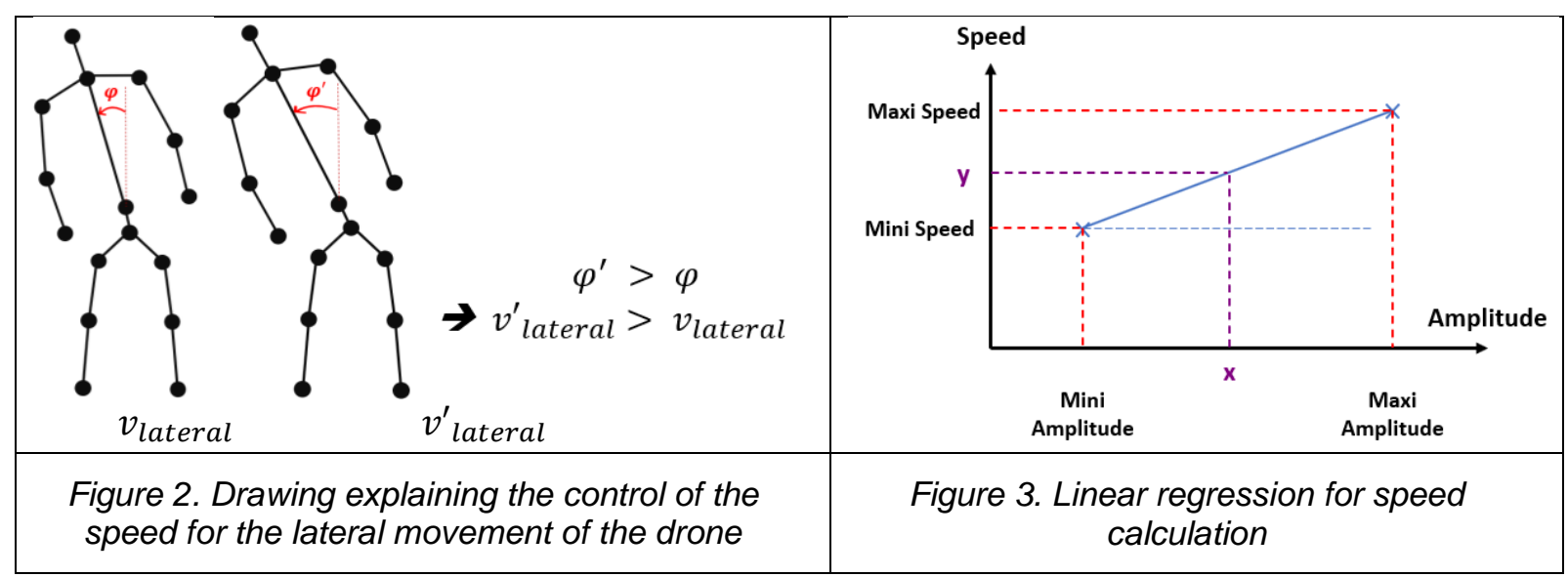

This principle, using the amplitude of movement to control the desired speed of the command, is applied to each command.

$$
m=\frac{\text { maxi Speed-mini Speed }}{\text { maxi Amplitude-mini Amplitude }} \quad ; \quad y=(x-\text { mini Amplitude }) \times m+\text { mini Speed }
$$

Where $m$ is the slope, $x$ the amplitude, and $y$ the calculated speed

\subsection{Design of a graphical user interface}

In addition to the development of drone piloting, it is important to provide feedback to the user. Drone's feedback is essential for effective use and has been implemented in a Graphical User Interface (GUI).

Additionally, one of the existing research gaps is the lack of additional functionalities other than the piloting itself. Thus, an extension of the functional capacity is studied and designed in this paper and is implemented through the Graphical User Interface.

\subsubsection{User feedback}

The most important element of feedback is the visual feedback from the drone's camera. It is essential to enable remote piloting when direct visual piloting is impossible. The Python library used for the piloting of the drone does not include this functionality. However, another library has recently been developed for the Tello drone and allows access to the video of the drone and has been used in this project (Hanker-lu, 2019). Thus, a new Python class was defined that communicates with the Tello drone by Wifi and displays the visual feedback on the user's screen.

Another feedback that was developed was the transcription on the screen of the Kinect view and skeleton detection. A small window is constantly displayed on the screen to allow the user to see the Kinect's field of view and ensure that the skeleton is detected. Controlling the drone with a body while receiving feedback from the system providing information about both location of the drone and the pilot's body form aims to make the interaction system more immersive than using the controllers.

Finally, it is important for the pilot to know the battery status and receive a warning when the battery level is low so that the drone can return or land timely. To do this, the user will have visual feedback on the battery percentage screen. An audible signal will be emitted at regular intervals if the battery level becomes low (less than 20\%) and at shorter intervals, if the level is very low (less than 10\%), to ensure the pilot is notified even if they are not looking at the screen.

\subsubsection{Menu for selection of standard drone functionality}

Another important objective of this research was to increase the number of commands that the user can send to the drone by using body gestures and conventional piloting, such as taking photos and videos or activating a specific functionality. To increase the number of commands without interfering with the driving quality, it was decided to use a GUI through an interactive menu where the user can choose the command to send.

To open this GUI, the user must raise their left arm in the air vertically for a fraction of a second. Once this movement has been performed, the menu appears on the screen. This gesture was chosen because it is simple to perform and is distinctly different from all other gestures that the system is looking for. Once the menu is opened with the left arm, the right arm will be used to select the command. The overall idea is to use the right arm like a clock hand and to turn it to choose a dial and thus a command. Thus, the 
centre of this menu, of the circle, will be virtually at the location of the user's right shoulder. Also, the display of the user's arm on the menu circle in real-time, through the use of the drawnow Python library (Python Software Foundation, 2020), has been developed to allow visual feedback of the movement and thus be sure of the chosen command. Icons are placed on the menu and allow the pilot to easily use these features from the first use without requiring training or memory exercise afterwards. Figure 4 presents the real-time interactive menu, and Figure 5 shows real-time skeleton detection.
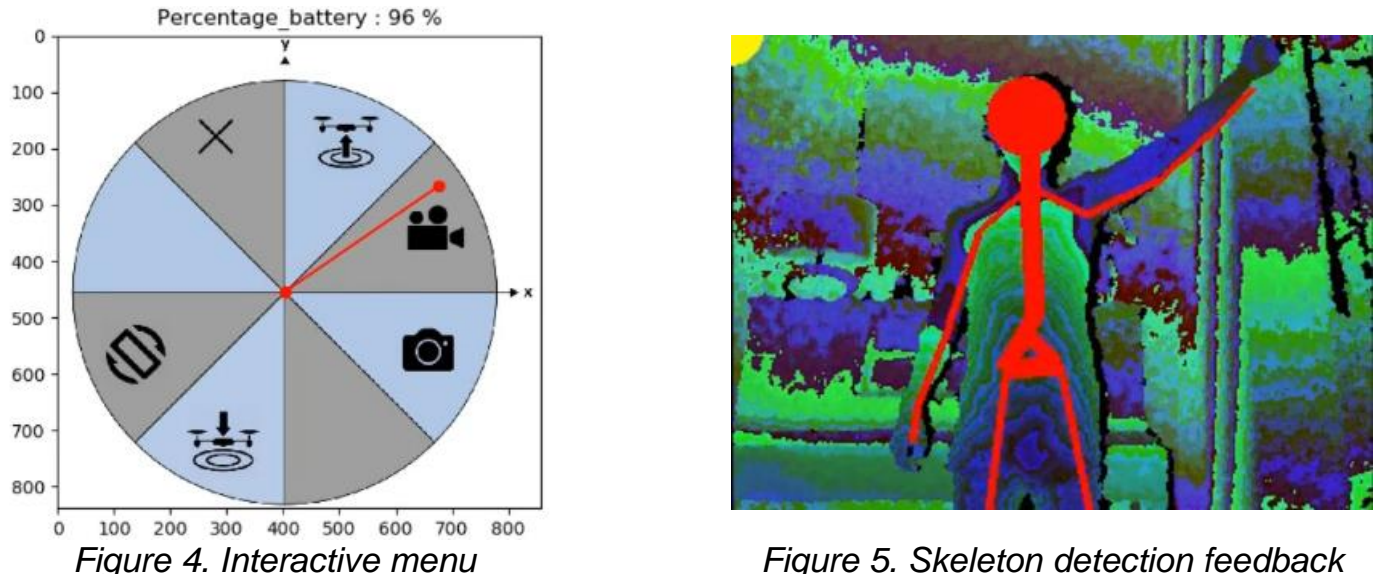

Figure 5. Skeleton detection feedback

When the menu is open, the drone goes into a stabilised hover while the command is being chosen. As suggested in Figure 4, the commands that will be implemented in this study will be the drone's takeoff and landing, video recording, picture taking, and flipping. However, other features can be implemented if necessary. The two areas without icons represent areas without commands. The one at the bottom right has been left without controls since it corresponds to the arms' natural position along the body and thus allows the user to give himself time to choose the control without feeling muscular fatigue. The top empty area is the most physically demanding area but could still be used if a new functionality needed to be added. The area with a cross closes the interactive menu without sending a command to resume the drone's classic piloting. Finally, in addition to the sound feedback presented earlier for the battery level, the exact percentage is displayed in the interactive menu.

\section{RESULTS}

To determine the success of the NUI, an experiment was designed with six participants. It aims to determine if the system could successfully identify the gestures and if the gestures could successfully control a drone's flight.

\subsection{Person recognition}

The movements designed and developed in this research were well recognised by the designed system and converted to drone commands. Figure 6, displays the experimental results in which 6 participants with different physical traits were successfully recognised and gestures recorded. This validates the recognition approach developed in this paper and demonstrates that it worked for all participants.
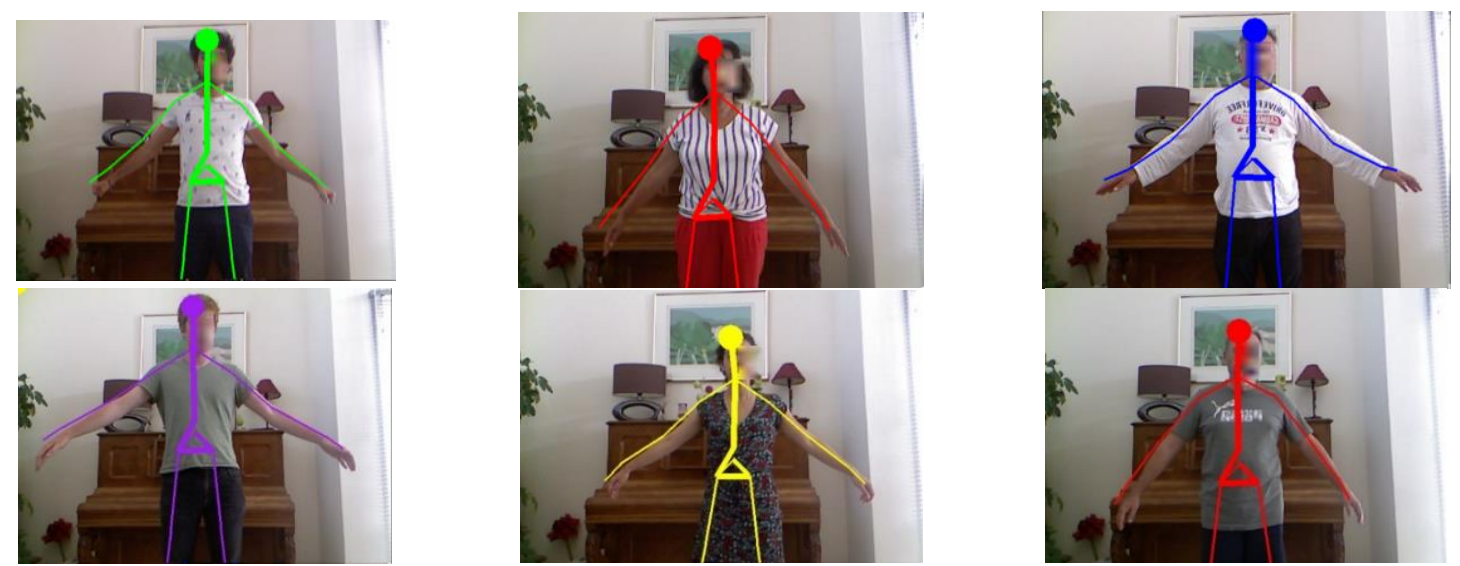

Figure 6. Gesture recognition tests for 6 subjects with different physical traits 


\subsection{Angle accuracy}

Experiments were carried out to evaluate the performance of the speed control and the accuracy of the angle calculation. To do this, a user was piloting the drone with the NUI developed in this paper while his movements were recorded with an external camera in front of him. The video allowed measurement of their body's angles when commands were sent to the drone and the desired speed. The speed commands sent to the drone were recorded and these two sets of values were compared. Figure 7 shows an example of the measurement of the real angle for the Right command. Several tests were realised for each command and in the end, the average error between the desired speed value and the speed value sent was less than $2 \%$. In addition, the measured value was decimal while the value sent to the drone was rounded off to the nearest integer, which means that the error was even smaller.

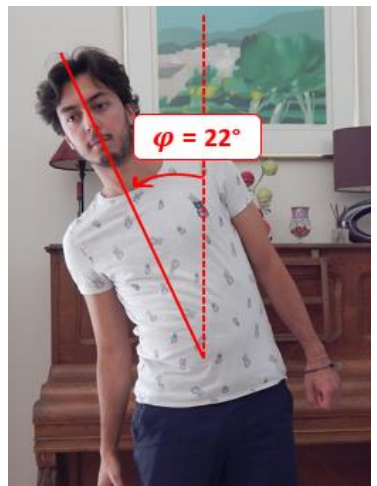

Measured Speed value measured: 43.1

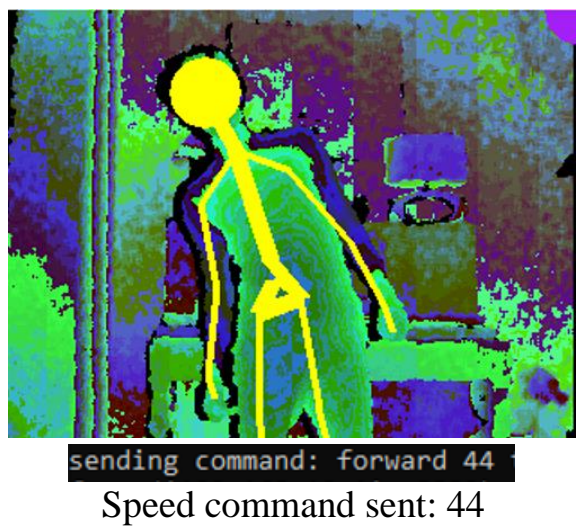

Speed command sent: 44

Figure 7. Speed command evaluation

\subsection{Flight tests}

To evaluate the piloting developed in this paper, a flight test was carried out. The experiment took into account the potential future use of this project and included piloting the drone, to the point of interest by avoiding some obstacles, taking a photo and returning to the starting point. To cover the professional and the amateur aspect, both experienced users and inexperienced users participated in this experiment. Video feedback of the embedded camera was provided on the screen in front of the user but since inexperienced users participated in the experiment, it took place in an open area where the user could always have visual feedback from the drone. To compare the gesture piloting solution of this project to the common piloting approach, the same experiment was carried out for each technique: body gesture and controller piloting.

Four people participated in the experiment. The first person was experienced with a classic controller and with the developed NUI. The second one was experienced only with traditional driving. The final two participants had never flown a drone before. The gesture commands were explained once, and a 30 -second training period was given to the users for each piloting technique. Each user conducted the task twice to record the data accurately. The results of this experiment are provided in Figure 8.

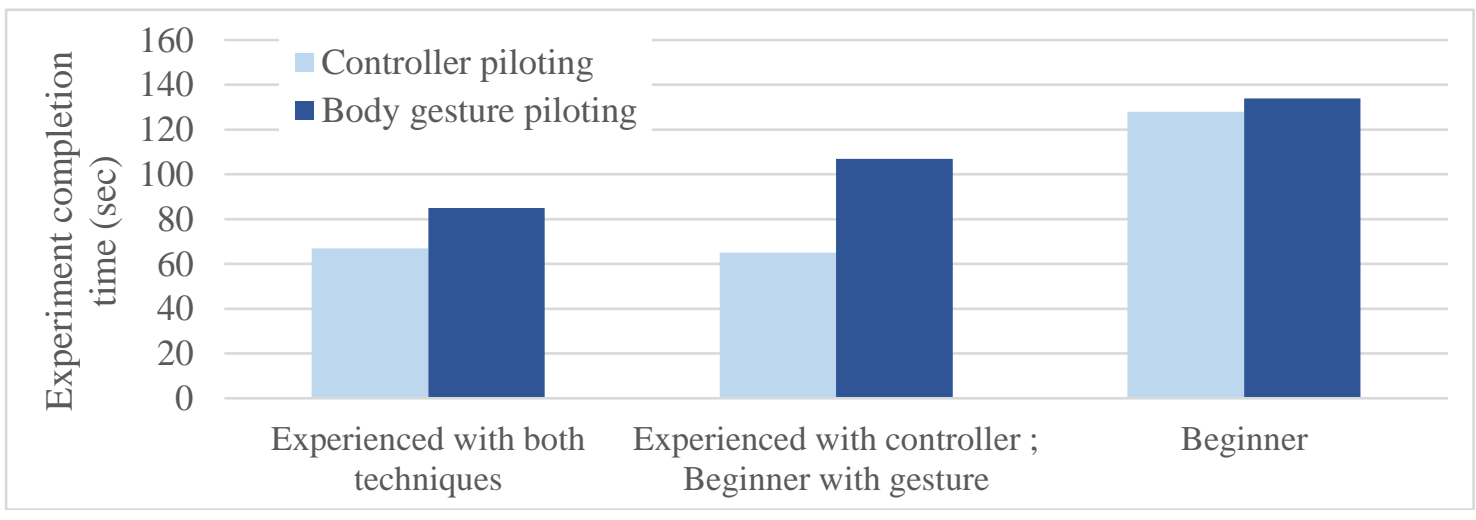

Figure 8. Experiment completion times for different types of user with each technique 


\section{DISCUSSION}

Several conclusions can be drawn from the flight test. All participants successfully carried out the experiment. Hence it can be asserted that the NUI developed throughout this research allows the piloting of a drone, and to carry out tasks such as visual inspection and photography.

In terms of performance time to complete the tasks, for a user experienced with both techniques, the experience with gesture piloting is on average about $27 \%$ longer. Although longer, the experiment's completion time using body gestures is of the same order of magnitude as using the classic controller. Also, it appears that a person experienced in controller flown drones will pilot a drone more efficiently using body gestures, than a person who is a total novice in drone piloting. It appears that the task completion times are similar between the two piloting techniques for an inexperienced person. A notable point underlined by this category of user is that piloting using body movements is intuitive for the majority of commands. After an explanation and a few seconds of handling, users did not ask for the gestures necessary to pilot the drone (except for the backward command) while inexperienced pilots asked several times about the controller's joystick commands. It appears that piloting with body movements of the developed system is more intuitive and natural than traditional piloting with a controller and joysticks as well as less mentally demanding for inexperienced users.

Changes in design could be made in the future. A non-linear model (e.g. exponential or logarithmic) could replace the linear model linking the amplitude of movement to the speed of flight currently in place. This would make it possible to have greater piloting precision at low speeds. Finally, more functionalities can be added by organising the interactive menu differently if a project requires it. Using gestures and the developed NUI to pilot a drone is natural which means that the user can concentrate less on piloting and more on the drone's visual feedback and the mission. This means they may carry out a better-quality intervention. The implementation of additional functionalities to piloting and flight speed control made in this paper allows reaching a complete NUI and realising both recreational and professional drone missions.

During piloting, a latency is felt between two orders that lengthens the mission's total completion time. This latency, of around 1.3 seconds, is mainly due to the Tello Drone SDK and could be drastically reduced by changing SDK or drone. This would make the task completion time faster and improve the performance of the system. Hence, the NUI would likely outperform the conventional controller for beginners, as the completion time is already similar and competitive for experienced users. The NUI presented in this paper represents an efficient alternative to drone piloting regarding the performance, but is more natural, immersive, and intuitive. Ease of use of the developed system could increase drones' use, make it more accessible, and contribute to the societal transformation in many sectors.

With the development of the prototype system detailed in this paper, future studies should consider the impact of experience on controlling both the controller input and body gesture input. Additionally, the latency and accuracy should be tested with all user groups, and real-world piloting should be demonstrated within experimental work.

\section{CONCLUSION}

In this work, a Natural User Interface allowing a user to pilot a drone with body gestures was designed and developed. The analysis of the gestures used to pilot the drone was done by calculating, in realtime, the angles created between the joints of the user's skeleton and by associating gestures to the angles obtained. A Graphical User Interface was developed during the project, providing different types of user feedback to ensure compatibility with future drone operators' envisaged needs. Thus, visual feedback from the drone's camera, skeleton detection and battery status have been set up. This work also fills some important gaps in the existing literature on the piloting of drones using gestures, such as the design of an interactive menu to offer functionalities other than just drone piloting e.g. taking off, landing, taking pictures and videos, and flipping. The control of the drone's flight speed represented another major innovative aspect and has also been developed in this research focusing on the amplitudes of movements. Finally, a user experiment was carried out and shows that the NUI developed during this project is intuitive, natural, and immersive, allowing accurate piloting of a drone for tasks such as transportation, visual inspection in remote or dangerous environments or for more general hobbyist tasks such as photography and videography. This research is a step towards the use of body gestures in fully piloting drones with all features and functionality, which could revolutionise the professional and hobbyist communities. 


\section{REFERENCES}

Abtahi, P., Y.Zhao, D., L.E, J. \& Landay, J. A., (2017). “Drone Near Me: Exploring Touch-Based Human-Drone Interaction", The Proceedings of the ACM on Interactive, Mobile, Wearable and Ubiquitous Technologies, September, No 34, pp. 8.

Al-Shamayleh, A.S., Ahmad, R., Abushariah, M.A., Alam, K.A., Jomhari, N., (2018). "A systematic literature review on vision based gesture recognition techniques" in Multimedia Tools and Applications 77.

Andujar, M. \& Tezza, D., (2019). “The State-of-the-Art of Human-Drone Interaction: A Survey,” in IEEE Access, vol. 7, pp. 167438-167454, 2019, doi: 10.1109/ACCESS.2019.2953900.

Ars Electronica, (2020). Humphrey II. [online], Available at: https://ars.electronica.art/futurelab/project/humphrey-ii/ (12 04 2020).

Baudouin-Lafon, T. B. a. M., (1993). "Remote Control of Objects Using Free-Hand Gestures", Charade, 36(7), pp. 28-35.

Cauchard, J. R., E, J. L., Zhai, K. Y. \& Landay, J. A., (2015). "Drone \& me: an exploration into natural humandrone interaction", Proceedings of the 2015 ACM International Joint Conference on Pervasive and Ubiquitous Computing.

Cherpillod, A., Mintchev, S. \& Floreano, D., (2016). "Embodied Flight with a Drone”, [online], Available at: https://arxiv.org/ftp/arxiv/papers/1707/1707.01788.pdf (10/11/2020)

Dante Tezz, M. A., (2019). "The State-of-the-Art of Human-Drone Interaction: A Survey”, IEEE Access.

DJI, (2020), "Tello". [online], Available at: https://store.dji.com/fr/shop/tello-series (15/11/2020).

Suarez-Fernandez, R., Sanchez-Lopez, J.L., Sampedro, C., Bavle, H. , Molina, M., and Campoy, P. (2016), "Natural user interfaces for human-drone multi-modal interaction", June 2016, International Conference on Unmanned Aircraft Systems (ICUAS), pp. 0.

Goodrich, M. A., \& Schultz, A. C. (2008). Human-robot interaction: a survey. Now Publishers Inc.

Hanker-lu, (2019). Tello_Video Python Library. [online] Available at: https://github.com/dji-sdk/TelloPython/tree/master/Tello_Video (22/07/2020).

Hasanuzzaman, M., Ampornaramveth, V., Zhang, T., Bhuiyan, M. A., Shirai, Y., \& Ueno, H. (2004). "Real-time vision-based gesture recognition for human robot interaction", IEEE International Conference on Robotics and Biomimetics, IEEE, pp. 413-418.

Mashood, A., Noura, H., Jawhar, I., \& Mohamed, N. (2015). “A gesture based kinect for quadrotor control”, International Conference on Information and Communication Technology Research (ICTRC) (pp. 298301). IEEE.

Microsoft, (2012). “Kinect Sensor” [online], Available at: https://docs.microsoft.com/en-us/previousversions/microsoft-robotics/hh438998(v=msdn.10)?redirectedfrom=MSDN (10/11/2020).

Natarajan, K., Nguyen, T. H. D., \& Mete, M. (2018). "Hand gesture controlled drones: An open source library", 1st International Conference on Data Intelligence and Security (ICDIS), IEEE, pp. (168-175).

Pfeil, K., Koh, S. L., \& LaViola, J. (2013). "Exploring 3d gesture metaphors for interaction with unmanned aerial vehicles", International conference on Intelligent user interfaces, pp. (257-266).

Pisharady, P.K., Saerbeck, M., (2015). "Recent methods and databases in vision-based hand gesture recognition: A review". Computer Vision and Image Understanding 141, 152-165.

Python Software Foundation, (2020). "Drawnow library". [online], Available at: https://pypi.org/project/drawnow/ (23/07/2020).

Rautaray, S.S., Agrawal, A., (2015). "Vision based hand gesture recognition for human computer interaction: a survey", Artificial intelligence review 43, 1-54.

Rognon, C., Mintchev, S., Dell'Agnola, F., Cherpillod, A., Atienza, D., \& Floreano, D. (2018). "Flyjacket: An upper body soft exoskeleton for immersive drone control", IEEE Robotics and Automation Letters, 3(3).

Sanna, A., Lamberti, F., Paravati, G., \& Manuri, F. (2013). “A Kinect-based natural interface for quadrotor control", Entertainment Computing, 4(3), 179-186.

Sarkar, A., Patel, K. A., Ram, R. G., \& Capoor, G. K. (2016). Gesture control of drone using a motion controller. International Conference on Industrial Informatics and Computer Systems (CIICS), IEEE, pp. (1-5).

Shotton, J., Fitzgibbon, A. \& Sharp, T., (2010). "Real-Time Human Pose Recognition in Parts from Single Depth Images", IEEE, CVPR 2011, pp. (1297-1304).

Stoica, A., Salvioli, F., \& Flowers, C. (2014), "Remote control of quadrotor teams, using hand gestures", Proceedings of the 2014 ACM/IEEE international conference on Human-robot interaction, pp. (296-297).

Stone, Z., (2017). "Review: The Hand-Controlled Aura Drone". [online], Available at: https://www.forbes.com/sites/zarastone/2017/11/20/review-the-hand-controlled-aura-drone-will-make-youjealous-of-your-kids/\#5fb9dfb85041 (03/05/2020).

Sun, T., Nie, S., Yeung, D. Y., \& Shen, S. (2017), "Gesture-based piloting of an aerial robot using monocular vision", IEEE International Conference on Robotics and Automation (ICRA), pp. (5913-5920).

Toumi, T., \& Zidani, A. (2014). "From human-computer interaction to human-robot social interaction", arXiv.

Ultraleap, (2019). “Leap Motion Controller”. [online], Available at:

https://www.ultraleap.com/datasheets/Leap_Motion_Controller_Datasheet.pdf, (02/05/2020). 